\title{
Is There a Link between Basal Metabolic Rate, Spleen Volume and Hepatic Growth Factor Levels in Patients with Obesity-Related NAFLD?
}

\author{
Giovanni Tarantino ${ }^{1, *}$, Vincenzo Citro ${ }^{2}$, Paolo Conforti ${ }^{3}$, Clara Balsano ${ }^{4}(\mathbb{D}$ and \\ Domenico Capone 5 \\ 1 Department of Clinical Medicine and Surgery, Federico II University Medical School of Naples, 80131 Napoli \\ NA, Italy \\ 2 Department of General Medicine, "Umberto I" Hospital, Nocera Inferiore (Sa), 84014 Nocera Inferiore SA, \\ Italy; v.citro@libero.it \\ 3 “Federico II" University Medical School of Naples, 80131 Napoli NA, Italy; pconforti8@gmail.com \\ 4 Department of Clinical Medicine, Life, Health \& Environmental Sciences-MESVA, University of L'Aquila, \\ 67100 L'Aquila AQ, Italy; clara.balsano@cc.univaq.it \\ 5 Care Department of Public Health and Drug-Use, Section of Medical Pharmacology and Toxicology, \\ “Federico II" University, 80131 Naples NA, Italy; docapone@unina.it \\ * Correspondence: tarantin@unina.it
}

Received: 31 July 2019; Accepted: 16 September 2019; Published: 20 September 2019

\begin{abstract}
Background: Recent pieces of research point to a link between basal metabolic rate (BMR) and non-alcoholic fatty liver disease (NAFLD) or hepatic steatosis (HS). The spleen in obese patients is associated with the cardiovascular system. Enlargement of the spleen is suggestive of nonalcoholic steatohepatitis (NASH). Patients with NASH present an increase in growth factor (HGF) as well as those with advanced heart failure. Interleukin-16 and interleukin-12p40 levels were found to correlate significantly with BMI, and waist circumference. Aim: We tried to find a relationship between BMR, spleen length and HGF. Methods: We analysed retrospective data from 80 obese patients with NAFLD. We evaluated indices of indirect calorimetry by the bioimpendance analysis; carotid intima-media thickness (IMT), spleen length (SLD) and HS by ultrasonography; serum HGF, IL-16, IL-12p40 and IL-6 concentrations by a magnetic bead-based multiplex immunoassays and the severity of NAFLD by BARD score $>2$. Results: HGF levels of the obese were higher than those of controls, $p<0.001$. At linear regression, BMR was foreseen by spleen length $(p<0.001)$, which was predicted by HGF $(p=0.04)$. BMR was predicted by IL-16 $(p=0.005)$, which predicted HGF, $p=0.034$. Only fat mass, among other factors, predicted early atherosclerosis, $p=0.017$; IL-12p40 did not predict IMT, HGF and BMR ( $p=0.57,0.09$ and 0.59 , respectively). The BARD score $>2$ was negatively predicted by BMR and FFM ( $p=0.032$ and 0.031 , respectively), at the logistic regression. Interesting findings at the extended regression (mediation effect) were: IL-16 was likely causal in predicting BMR by HGF levels; HGF was influential in predicting BMR by SLD level. HS was predicted by SLD in males $(p=0.014)$, of advanced age $(p<0.001)$ and by BMR $(p<0.001)$. IL- 6 concentrations, but not BMR were influential in the prediction of HS by SLD. Conclusion: These data reinforce the concept that the immune system is a sensor of the metabolic state, showing a link between HGF levels and BMR, which is mediated by IL-16 (cytokine inducing a cascade of inflammatory factors), and ascertaining the influential effect of the spleen, as main immune organ.
\end{abstract}

Keywords: NAFLD; obesity; HGF; calorimetry indices; spleen 


\section{Introduction}

There is a wide consensus about the immune system as a sensor of the metabolic state [1]. In a pioneering study, obese subjects showed lower resting metabolic rate (RMR) than control subjects [2], but recently this finding is challenged [3]. In recent year, RMR has been substituted by the more precise basal metabolic rate (BMR), which depends on body composition as expressed by fat-free mass (FFM) and fat mass (FM) [4,5]. In this context, increased BMR may be a clue of MS when dealing with hepatic steatosis (HS) or nonalcoholic fatty liver disease (NAFLD) patients [6]. Furthermore, a regression of NAFLD following improvement of the increased BMR was clearly showed [7].

Novel results highlight the important contribution that spleen has on resting energy expenditure (REE), synonym of RMR [8]. Spleen, was found enlarged at significant levels (38\%) in obese patients [9]. Indeed, splenic enlargement is well detected by ultrasonography as length of the organ [10]. The association between spleen and cardio vascular (CV) system is tested by multiple studies, providing evidence of a cardio-splenic axis in humans [11-14].

Similarly, a liver-spleen axis has been recently proposed in the sense that increased spleen volume-a stable index of chronic inflammation and activation of the immune system-is suggestive of advanced HS [15,16]. Hepatic growth factor (HGF), secreted by mesenchymal cells, has one of its expressions in the spleen [17], acting via both a paracrine and an endocrine mechanism [18], specifically through its interaction with c-met receptor as regulator of immune cell functions [19]. HGF plays a role in regulating the chemotaxis of T cells into heart tissue [20-22]. Still, plasma from patients with advanced heart failure presents elevated levels of HGF [23]. On the other hand, patients with the most severe form of NAFLD, i.e., nonalcoholic steatohepatitis (NASH) present a significant increase in circulating levels of HGF [24].

Authors found that the increased levels of $f$ interleukin-(IL)-16 and those of IL-12p40 correlated significantly with BMI, and waist circumference (WC) [25]. Northern blotting has identified IL-16 mRNA predominantly in spleen. [26]. In this milieu IL-12p40 could induce the expression of IL-16 [27,28]. At the best of our knowledge, no research raised a question as to whether BMR can actually be linked to some immune system parameters such as spleen as well as to L-16, IL-12p40 and HGF in obesity-related NAFLD patients. Similarly, there are no literature data on the relationship between atherosclerosis, evaluated as carotid intima-media thickness (IMT) and the afore mentioned factors of immunity and/or indirect calorimetry indices. As key aim, we tried to verify whether the correlation between the NAFLD severity and spleen was mediated by IL-6, a mediator of a number of chronic inflammatory conditions.

Pursuing the objective of clarifying these aspects, at the light of the previously cited studies, which permitted somehow to hypothesise this intriguing association, we analysed in a retrospective way data from obese patients with low prevalence of co-morbidities, but NAFLD.

\section{Method}

\subsection{Patients}

We carried out a cross-sectional type of observational (retrospective) study where at a particular point of time we analysed characteristics of obese patients without follow-up, using as main variables: HGF, IL-16 and IL-12p40 levels, IL-6 concentrations, spleen length, IMT values, indirect calorimetry indices and HS scores. Specifically, this sub-study used the same original patient sample contained in a previous research [29], but with completely different analytical/statistical approaches resulting to be equally valid, [29].

\subsection{Inclusion Criteria}

Eighty obese patients of different grade of obesity, on calorie-reduced, low-fat diet and sedentary lifestyle, with low prevalence of co-morbidities, such as type 2 diabetes mellitus and hypertension but NALFD, US-documented, were retrospectively re-evaluated. 


\subsection{Exclusion Criteria}

We excluded patients from this recent evaluation if, at the time of blood specimen collection, they had self-reported present or antecedent unexplained muscle trauma in the past months or minor/major surgery or other chronic disease, such cancer or cardiovascular events or H. pylori infection (IL-16) or lung fibrosis (HGF) and the use of medications that might have affected body composition or muscle metabolism (e.g., steroids) or could have affected inflammation response.

Furthermore, viral, autoimmune, metabolic liver diseases (Wilson disease, hemochromatosis or antitrypsin deficiency) were ruled out by using appropriate testing, according to well-accepted diagnostic guidelines. Celiac disease was excluded by evaluating IgA anti-tissue transglutaminase antibodies. Alcohol abuse was disallowed, following the DSM-IV diagnostic criteria, by means of screening tests such as MAST (Michigan Alcohol Screening Test) and CAGE (Cut down, Annoyed, Guilty, and Eye opener), as well as random tests for blood alcohol concentration and the use of a surrogate marker, e.g., Mean Corpuscular Volume. Patients on anti-hypertensive drugs, and those treated with metformin or insulin, maintained a balanced therapeutic regimen throughout the study.

\subsection{Anthropometric Evaluation}

We established the three classes of obesity (light, moderate, and severe or I-II-III) on the basis of BMI cut-off points of 30-34.9 and 35-39.9 and $>40 \mathrm{~kg} / \mathrm{m}^{2}$, respectively.

Visceral obesity was identified by measuring WC at the midpoint between the lower border of the rib cage and the iliac crest. Hip circumference was measured around the widest part of the buttocks, with the tape parallel to the floor, and the waist-to-hip ratio (WHR) was calculated.

\subsection{Metabolic Assessment}

The Adults Treatment Panel III was selected to ascertain the presence of the metabolic syndrome (MS), considering at least three criteria: plasma glucose concentrations $\geq 100 \mathrm{mg} \mathrm{dL}^{-1}, \mathrm{WC}>102 / 88 \mathrm{~cm}$ (male/female), serum HDL concentration $<50 \mathrm{mg} \mathrm{dL}^{-1}$ for women and $<40 \mathrm{mg} \mathrm{dL}^{-1}$ for men, blood pressure $\geq 130 / 85 \mathrm{mmHg}$, and serum triglyceride concentration $\geq 150 \mathrm{mg} \mathrm{dL}^{-1}$. Furthermore, we applied the criteria for Europids following the International Diabetes Classification (IDF). Accordingly, patients were defined as having the metabolic syndrome if they showed central obesity (measured as WC with ethnicity specific values, e.g., for males and females equal or superior to 94 and $80 \mathrm{~cm}$, respectively), plus any two of the following four factors: Triglycerides $>150 \mathrm{mg} / \mathrm{dL}$ or specific treatment for this lipid abnormality; cholesterol HDL $<50 \mathrm{mg} / \mathrm{dL}$ for females and $40 \mathrm{mg} / \mathrm{dL}$ for males or specific treatment for this dyslipidemia; systolic and diastolic blood pressure equal or superior to 130 and $85 \mathrm{mmHg}$, respectively; fasting plasma glucose $>100 \mathrm{mg} / \mathrm{mL}$ or previously diagnosed type 2 diabetes mellitus. International Diabetes Federation, 2007; IR was evaluated by the HOmeostatic Metabolic Assessment (HOMA) method employing the formula: fasting insulin $(\mu \mathrm{U} / \mathrm{mL}) *$ fasting glucose $(\mathrm{mg} / \mathrm{dL}) / 405[30]$.

\subsection{Laboratory Data}

HGF, IL-6, Il-16 and IL12p40 levels of 78 patients derived from a previously studied 48-cytokine/chemokine panel [31], which was performed on serum samples using a magnetic bead-based multiplex immunoassays (Bio-Plex) (BIO-RAD Laboratories, Milano, Italy) following manufactures' instructions. Data from the reactions were acquired using the Bio-Plex 200 reader, while a digital processor managed data output and the Bio-Plex Manager software returned data as Median Fluorescence Intensity (MFI) and concentration (pg/mL).

HGF levels of the obese were compared to those of 33 controls.

To assess liver fibrosis, we used the Bard score. In brief, three variables were combined in a weighted sum (BMI $>$ or $=28=1$ point, AST/ALT ratio of $>$ or $=0.8=2$ points, $\mathrm{T} 2 \mathrm{DM}=1$ point) to form an easily calculated composite score for predicting advanced fibrosis. A score of $2-4$ was associated 
with an OR for advanced fibrosis of 17 (confidence interval 9.2 to 31.9) and a negative predictive value of $96 \%$ [32]. The hs-CRP values, determined by the ELISA test, with reference values between 0.03 and $0.86 \mathrm{mg} / \mathrm{dL}$ in healthy men and between 0.02 and $0.91 \mathrm{mg} / \mathrm{dL}$ in healthy women (BioCheck, Inc., San Francisco, CA, USA) were assayed in collected and aliquoted samples and frozen at $-20^{\circ} \mathrm{C}$.

\subsection{Ultrasound Features}

Ultrasound (US) measurements were obtained by an Esaote (Genoa, Italy) system.

We chose the spleen length, assessed as spleen longitudinal diameter (SLD)-measured on longitudinal scan and carried out by the postero-lateral approach-to evaluate the spleen volume. The classification of "bright liver" or hepatic steatosis (HS) was based on the following scale of hyperechogenity: grade $0=$ absent, $1=$ light, $2=$ moderate, $3=$ severe, pointing out the difference between the densities of the liver and the right kidney [33], using a Convex Probe, with access to the liver through intercostal spaces along the mid-axillary line.

Visceral Adipose Tissue (VAT), was evaluated at Ultrasound and defined as the distance between the anterior wall of the aorta and the internal face of the recto-abdominal muscle perpendicular to the aorta, measured one $\mathrm{cm}$ above the umbilicus (when the aortic walls were obscured by bowel gas, a Doppler scan was used to detect VAT) [34].

IMT was defined as the distance between the ultrasound interfaces of the lumen-intima and media-adventitia. Six manual measurements were performed, with automatic border detection, at equal distances along one $\mathrm{cm}$ on the far wall of the common carotid, according to the consensus statement from the American Society of Echocardiography Carotid Intima-Media Thickness Task Force, endorsed by the Society for Vascular Medicine [35].

\subsection{Indirect Calorimetry}

RMR was measured by indirect calorimetry using a canopy system (V max $29 \mathrm{~N}$, Sensor Medics, Anaheim, CA, USA) in a quiet environment and with patients in the supine position for $30 \mathrm{~min}$ before computation. After a 15-20 min adaptation to the instrument, oxygen consumption and carbon dioxide production were determined for $45 \mathrm{~min}$. Energy expenditure was derived from $\mathrm{CO}_{2}$ production and $\mathrm{O} 2$ consumption by the means of the Weir formula neglecting protein oxidation [36]. BMR, expressed as $\mathrm{kcal} / 24 \mathrm{~h}$, was adjusted for changes in FFM, which was evaluated by single-frequency bioimpedance analysis (BIA) obtaining a RMR/FFM ratio, expressed as kcal/24 $\mathrm{h}^{*} \mathrm{~kg}$ of body. FM and FFM percentage were estimated using the device's standard built in prediction equations and were displayed on the machine and printed out [6].

BIA evaluation was performed between 10:00 a.m. and 4:00 p.m. The participants were required to fasting and avoiding vigorous exercise for at least $1 \mathrm{~h}$ before BIA assessment. BIA measurements were performed while patients stood barefoot on the metal surface of the device and kept their arms loose and in parallel with the body. Measurement took 1-2 $\min (\mathrm{s})$ for each patient, and results were automatically printed out from the device. FM, FFM, and total body water were measured by BIA (6). The measurements were recorded by well-trained staff, using a BIACorpus RX 4000 (Medi-Cal Healthcare GmbH, Karlsruhe, Germany).

\subsection{Statistics}

Data, derived from a normally distributed population, were given as mean plus SD. Variables not normally distributed or ordinals are expressed as median plus 25-75 interquartile range (IQR). The difference in medians was assessed by the Mann-Whitney test.

Frequency tables and multinomial logistic regression was used to assess relationships between obesity classes and MS presence.

To study predictions, we carried out various types of regression techniques. The linear regression analysis (ordinary least squares or OLS) as univariate analysis was employed evaluating the coefficient with its standard error, 95\% confidence intervals (CI), the $t(t-v a l u e)$ and squared-R. An ordered 
probit model was arranged to estimate predictions between an ordinal dependent variable, i.e., HS at US, expressed as severity grades (1-3) and a set of independent variables. The output showed the coefficients, their standard errors, the z-statistic (also called a Wald z-statistic), and the associated $p$-values. In suspicion of heteroscedasticity and having detected the presence of few outliers, we analyzed the correlation by the robust regression, using Least Absolute Deviations (LAD) Regression.

Remaining unclear whether prediction represents unidirectional or bidirectional causality, to try to partially overcome this aspect, endogenous variables were studied.

A variable is said to be endogenous within the causal model if its value is determined or influenced by one or more of the independent variables, [37]. A purely endogenous variable is a factor that is entirely determined by the states of other variables in the system. An extended regression that fits a linear regression model that accommodates any combination of endogenous covariates was run ad hoc to test a possible mediation effect. Still, we evaluated at multiple linear regression the so called $\beta$ or Beta factor, in order to study which of the independent variables have a greater effect on the dependent variables.

Dealing with a binary dependent variable the prediction tool carried out was the logistic regression by which the Odds ratio with related $95 \% \mathrm{CI}$ was evaluated, using as categorical variable BARD $>$ or $<2$.

In order to evaluate the degree of pair observations at US, we adopted the concordance correlation coefficient $\left(\rho_{\mathrm{c}}\right)$, which measures precision and accuracy.

The power of this study was calculated on the difference of means of HGF levels between the obese and control group. To further deepen this aspect, a power analysis was performed using a slope test in the linear regression between the HS (d.v.) levels and the SLD (i.v.) values in the whole population of obese patients.

Stata15.1 was the program on which we run statistics.

\section{Results}

\subsection{Prevalence}

First of all, the median HGF levels of the obese population resulted to be higher than that of 33 controls, i.e., 491 (425-565) versus 230 (136-278) pg/mL; two-sample Wilcoxon rank-sum (Mann-Whitney) test $p<0.001$, Figure 1. Concentrations of HGF in our controls were quite similar to the reported Biorad normal values, i.e., median 195, [38].

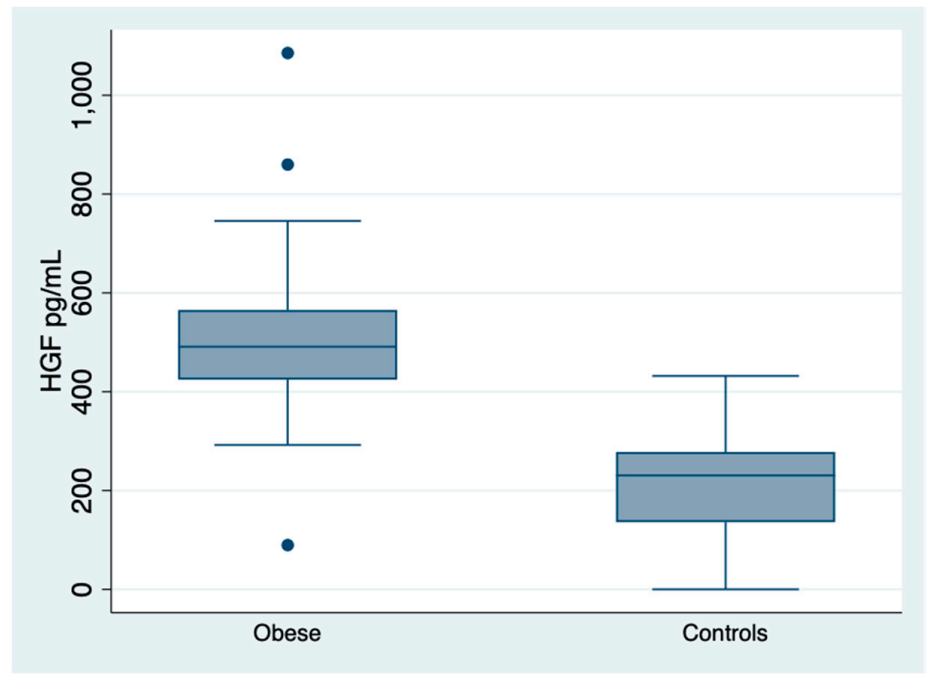

Figure 1. Growth factor concentrations in the obese and controls. Legend to Figure 1: HGF, hepatic growth factor. It is noteworthy to stress that the outliners were very few and that there is scarce overlapping between groups. 
The obese population was characterised by normal or slightly elevated liver enzymes, mainly confronting the values of ALT (key enzyme in NAFLD patients) to the healthy upper limits, evidenced in [39]. Other clinical, laboratory and instrumental characteristics are shown in Table 1.

Table 1. Main characteristics of the obese population.

\begin{tabular}{cccc}
\hline Age (Years) & $\mathbf{4 6}$ (34-53) & $\begin{array}{c}\text { Gender } \\
\text { Males/Females } \boldsymbol{n}\end{array}$ & $\mathbf{3 6 / 4 4}$ \\
\hline Obesity classes I/II/III $\mathrm{n}$ & $8 / 26 / 46$ & $\mathrm{CRP}$ & $0.56(0.27-1.3)$ \\
MS (APT III) yes/not & $51 / 29$ & HGF pg/mL & $491(425-565)$ \\
MS (ID) yes/not & $51 / 29$ & IL-12p40 pg/mL & $234(130-317)$ \\
HS at US grade1/2/3 & $22 / 50 / 8$ & IL-6 pg/mL & $5.7(2.3-17-5)$ \\
BARD > 2 Males/Females $\mathrm{T}$ & $9 / 27$ & T2DM n & 18 \\
SLD at US cm & $11(10.1-12.4)$ & HOMA & $2.75(1.9-4.7)$ \\
BMR kcal/24h & $2220(1770-2439)$ & Hypertension n & 4 \\
FFM \% & $56(49-67)$ & Cholesterol mg/dL & $191 \pm 36$ \\
FM \% & $52 \pm 7.5$ & IMT mm & $0.9(0.7-1.1)$ \\
RMR/FFM kcal/24 h*kg of body & $39(34-43)$ & PChe U/L & $9605(8300-10876)$ \\
ALT (Males) U/L & $36.5(27-41)$ & ALT (Females) U/L & $23(17-31)$ \\
AST (Males) U/L & $24(21-27)$ & AST (Females) U/L & $20(16-23)$ \\
G-GT mu/L & $25(16-42)$ & AP mu/L & $73(61-91.5)$ \\
\hline
\end{tabular}

Legend to Table 1: MS, metabolic syndrome; HS at US, hepatic steatosis at ultrasonography; SLD, spleen longitudinal diameter; BMR, basal metabolic rate; FFM, free fat mass; FM, fat mass; RMR, resting metabolic rate; HGF, hepatic growth factor; IMT, carotid intima-media thickness; PChe, pseudo cholinesterase; AP, alkaline phosphatase; G-GT, gamma-glutamyl transpeptidase; AST/ALT, transaminases; CRP; C reactive protein; T2DM, type 2 diabetes mellitus.

There was no difference in frequency when relating obesity classes to MS presence, Table 2.

Table 2. Relationships between obesity classes and metabolic syndrome (MS) presence.

\begin{tabular}{|c|c|c|c|c|c|c|}
\hline Frequency Table & \multicolumn{3}{|c|}{ Pearson Chi-Squared $=4.838$} & \multicolumn{3}{|c|}{$\operatorname{Pr}=0.089$} \\
\hline \multirow[t]{2}{*}{ Obesity class } & \multicolumn{6}{|c|}{ MS } \\
\hline & & Not & Yes & \multicolumn{3}{|c|}{ Total } \\
\hline \multirow[t]{2}{*}{$\mathbf{I}$} & $\mathrm{n}$ & 4 & 4 & \multicolumn{3}{|c|}{8} \\
\hline & $\%$ & 13.79 & 7.84 & \multicolumn{3}{|c|}{10.00} \\
\hline \multirow[t]{2}{*}{ II } & $\mathrm{n}$ & 13 & 13 & \multicolumn{3}{|c|}{26} \\
\hline & & 44.83 & 25.49 & \multicolumn{3}{|c|}{46} \\
\hline \multirow[t]{2}{*}{ III } & $\mathrm{n}$ & 12 & 34 & \multicolumn{3}{|c|}{32.50} \\
\hline & $\%$ & 41.3 & 66.67 & \multicolumn{3}{|c|}{57.50} \\
\hline Total & $\mathrm{n}$ & 29 & 51 & \multicolumn{3}{|c|}{80} \\
\hline \multicolumn{7}{|c|}{ Multinomial logistic regression } \\
\hline \multicolumn{2}{|c|}{ Number of obs $=80$} & \multicolumn{5}{|c|}{ Prob $>$ chi-squared $=0.089$} \\
\hline d.v. & Obesity class & Coef. & Std. Err. & $z$ & $p>|z|$ & $\begin{array}{l}\text { (95\% Conf. } \\
\text { Interval) }\end{array}$ \\
\hline \multicolumn{7}{|l|}{ I } \\
\hline $\begin{array}{l}\text { i.v. } \\
\text { II }\end{array}$ & MS & -1.041 & 0.782780 & -1.33 & 0.183 & $-2.576 / 0.493$ \\
\hline $\begin{array}{l}\text { i.v. } \\
\text { III }\end{array}$ & $\begin{array}{c}\text { MS } \\
\text { (base outcome) }\end{array}$ & -1.041 & 0.516 & -2.02 & 0.044 & $-2.053 / 0.029$ \\
\hline
\end{tabular}

Concerning the main inflammatory responses, the median CRP concentration was $0.56(0.27-1.30)$ $\mathrm{mg} / \mathrm{L}$, specifically in females $0.55(0.34-1.38)$ and males 0.59 (0.23-1.22), being only the third quartile superior the normal range values. 


\subsection{Predictions}

The first step was to find any association between calorimetric indices and some parameters of immune system. The results evidenced a link between the spleen volume, assessed as its length, and both BMR and FFM. Furthermore, IL-16 concentrations predicted BMR, Table 3.

Table 3. Prediction of indices of indirect calorimetry by spleen longitude diameter (SLD). Prediction of basal metabolic rate (BMR) by IL-6 and IL-12p40 levels.

\begin{tabular}{|c|c|c|c|c|c|}
\hline \multicolumn{6}{|c|}{ Linear Regression, Robust } \\
\hline \multirow{3}{*}{$\begin{array}{c}\text { Number of obs }=\mathbf{8 0} \\
\text { d.v. BMR } \\
\text { i.v. SLD }\end{array}$} & \multicolumn{5}{|c|}{$R$-squared $=0.271$} \\
\hline & Coef. & Std. Err. & $t$ & $p>|t|$ & (95\% Conf. Interval) \\
\hline & 144.508 & 27.801 & 5.20 & $<0.001$ & 89.161/199.855 \\
\hline \multicolumn{6}{|c|}{ Linear Regression, Robust } \\
\hline Number of obs $=80$ & \multicolumn{5}{|c|}{$R$-squared $=0.143$} \\
\hline d.v. FFM & Coef. & Std. Err. & $t$ & $p>|t|$ & (95\% Conf. Interval) \\
\hline i.v. SLD & 3.348 & 1.146 & 2.92 & 0.005 & $1.068 / 5.60$ \\
\hline \multicolumn{6}{|c|}{ Linear Regression, Robust } \\
\hline Number of obs $=80$ & \multicolumn{5}{|c|}{$R$-squared $=0.006$} \\
\hline d.v. RMR/FFM & Coef & Std. Err. & $t$ & $p>|t|$ & ( $95 \%$ Conf. Interval) \\
\hline i.v. SLD & 0.3519 & 0.496 & 0.71 & 0.480 & $-0.636 / 1.339$ \\
\hline \multicolumn{6}{|c|}{ Linear Regression, Robust } \\
\hline Number of obs $=80$ & \multicolumn{5}{|c|}{$R$-squared $=0.005$} \\
\hline d.v. FF & Coef. & Std. Err. & $t$ & $p>|t|$ & (95\% Conf. Interval) \\
\hline i.v. SLD & -0.357 & 0.503 & -0.71 & 0.479 & $-1.358 / 0.643$ \\
\hline \multicolumn{6}{|c|}{ Linear Regression, Robust } \\
\hline Number of obs $=78$ & \multicolumn{5}{|c|}{$R$-squared $=0.089$} \\
\hline d.v. BMR & Coef. & Std. Err. & $t$ & $p>|t|$ & (95\% Conf. Interval) \\
\hline i.v. IL-16 & 2.117 & 0323677 & 2.89 & 0.005 & $0.658 / 3.576$ \\
\hline \multicolumn{6}{|c|}{ Linear Regression, Robust } \\
\hline Number of obs $=78$ & & & $R$-squa & .006 & \\
\hline d.v. BMR & Coef. & Std. Err. & $t$ & $p>|t|$ & (95\% Conf. Interval) \\
\hline i.v. IL-2p40 & -0.242 & 0.444 & -0.55 & 0.587 & $-1.127 / 0.642$ \\
\hline
\end{tabular}

Legend to Table 3: FF, fat mass; FFM, free fat mass; RMR, resting metabolic rate; RMR/FFM, resting metabolic rate/free fat mass ratio; SLD, spleen longitudinal diameter at ultrasonography. The low R-squared, in presence of significance, shows that even noisy, high-variability data can have a significant trend, even though data points fall further from the regression line in graph; d.v., dependent variable; i.v., independent variable. In bold are highlighted the significant ones.

A further step consisted in discovering any correlation between the early atherosclerosis, evaluated as IMT, and immune system parameters and indirect calorimetry indices taken into account in this study. As expected the role of adiposity was clearly evidenced in the sense that IMT was predicted by FF, Table 4. 
Table 4. Prediction of intima-media thickness (IMT) by SLD, fat-free mass (FFM), BMR, FF, fat-free mass (FFM), resting metabolic rate (RMR)/FFM ratio, IL-16 and IL-12p40.

\begin{tabular}{|c|c|c|c|c|c|}
\hline \multicolumn{6}{|c|}{ Linear Regression, Robust } \\
\hline \multirow{3}{*}{$\begin{array}{c}\text { Number of obs }=\mathbf{8 0}, \\
\text { d.v. IMT } \\
\text { i.v. SLD }\end{array}$} & \multicolumn{5}{|c|}{$R$-squared $=0.003$} \\
\hline & Coef. & Std. Err. & $t$ & $p>|t|$ & (95\% Conf. Interval) \\
\hline & -0.001 & 0.002 & -0.54 & 0.594 & $-0.00 / 0.003$ \\
\hline \multicolumn{6}{|c|}{ Linear Regression, Robust } \\
\hline Number of OBS $=80$ & \multicolumn{5}{|c|}{$R$-squared $=0.007$} \\
\hline d.v. IMT & Coef. & Std. Err. & $t$ & $p>|t|$ & (95\% Conf. Interval) \\
\hline i.v. FFM & 0.0002 & 0.0003 & 0.72 & 0.473 & $-0.0002 / 0.008$ \\
\hline \multicolumn{6}{|c|}{ Linear Regression, Robust } \\
\hline Number of obs $=80$ & \multicolumn{5}{|c|}{$R$-squared $=0.0002$} \\
\hline d.v. IMT & \multirow{2}{*}{$\begin{array}{l}\text { Coef. } \\
-1.09 \times \\
10^{-6}\end{array}$} & Std. Err. & $t$ & $p>|t|$ & (95\% Conf. Interval) \\
\hline i.v. MBR & & $8.58 \times 10^{-6}$ & -0.13 & 0.899 & $-0.00001 / 0.00002$ \\
\hline & \multirow{2}{*}{\multicolumn{5}{|c|}{$\begin{array}{l}\text { Linear Regression, Robust } \\
\qquad \text {-squared }=0.079\end{array}$}} \\
\hline Number of obs $=80$ & & & & & \\
\hline IMT & Coef. & Std. Err. & $t$ & $p>|t|$ & (95\% Conf. Interval) \\
\hline $\mathrm{FF}$ & 0.001 & 0.0005 & 2.43 & 0.017 & $0.0002 / 0.002$ \\
\hline \multicolumn{6}{|c|}{ Linear Regression, Robust } \\
\hline Number of obs $=80$ & \multicolumn{5}{|c|}{$R$-squared $=0.015$} \\
\hline IMT & Coef. & Std. Err. & $t$ & $p>|t|$ & (95\% Conf. Interval) \\
\hline RMR/FFM & -0.0006 & 0.0005 & -1.26 & 0.211 & $-0.001 / 0.0003$ \\
\hline \multicolumn{6}{|c|}{ Linear Regression, Robust } \\
\hline Number of obs $=78$ & \multicolumn{5}{|c|}{$R$-squared $=0.009$} \\
\hline d.v. IMT & Coef. & Std. Err. & $t$ & $p>|t|$ & (95\% Conf. Interval) \\
\hline i.v. HGF & 0.00002 & 0.00004 & 0.70 & 0.488 & $-0.00005 / 0.0001$ \\
\hline \multicolumn{6}{|c|}{ Linear Regression, Robust } \\
\hline Number of obs $=78$ & \multicolumn{5}{|c|}{$R$-squared $=0.0005$} \\
\hline d.v. IMT & Coef. & Std. Err. & $t$ & $p>|t|$ & (95\% Conf. Interval) \\
\hline i.v. IL-16 & 0.00001 & 0.00005 & 0.26 & 0.796 & $-0.00008 / 0.0001$ \\
\hline \multicolumn{6}{|c|}{ Linear Regression, Robust } \\
\hline Number of obs $=78$ & & & $R$-squa & 005 & \\
\hline d.v. IMT & Coef. & Std. Err. & $t$ & $p>|t|$ & (95\% Conf. Interval) \\
\hline i.v. IL-2p40 & -0.00002 & 0.00003 & -0.58 & 0.566 & $-0.00008 / 0.00004$ \\
\hline
\end{tabular}

Legend to Table 4: FF, fat mass; FFM, free fat mass; RMR, resting metabolic rate; RMR/FFM, resting metabolic rate/free fat mass ratio; SLD, spleen longitudinal diameter at ultrasonography; IMT, carotid intima-media thickness; d.v., dependent variable; i.v., independent variable. In bold is highlighted the significant one.

Homing in on the concentrations of HGF, this growth factor was strongly associated with two main immune system parameters such as the spleen length and concentrations of IL-16, Table 5. 
Table 5. Prediction of SLD, IL-16 and IL-12p40 levels by HGF.

\begin{tabular}{|c|c|c|c|c|c|}
\hline \multicolumn{6}{|c|}{ Linear Regression, Robust } \\
\hline Number of obs $=78$ & \multicolumn{5}{|c|}{$R$-squared $=0.086$} \\
\hline d.v. SLD & Coef. & Std. Err. & $t$ & $p>|t|$ & (95\% Conf. Interval) \\
\hline i.v. HGF & 0.003 & 0.002 & 2.09 & 0.04 & $0.0001 / 0.006$ \\
\hline \multicolumn{6}{|c|}{ Linear Regression, Robust } \\
\hline Number of obs $=78$ & \multicolumn{5}{|c|}{$R$-squared $=0.095$} \\
\hline d.v. HGF & Coef. & Std. Err. & $t$ & $p>|t|$ & (95\% Conf. Interval) \\
\hline i.v. IL-16 & 0.680 & 0.315 & 2.16 & 0.034 & $0.052 / 1.307$ \\
\hline \multicolumn{6}{|c|}{ Linear Regression, Robust } \\
\hline Number of obs $=78$ & \multicolumn{5}{|c|}{$R$-squared $=0.060$} \\
\hline d.v. HGF & Coef. & Std. Err. & $t$ & $p>|t|$ & (95\% Conf. Interval) \\
\hline i.v. IL-12p40 & 0.234 & 0.137 & 1.71 & 0.091 & $-0.038 / 0.507$ \\
\hline
\end{tabular}

Legend to Table 5: HGF, hepatic growth factor; SLD, spleen longitudinal diameter at ultrasonography; IL-16, interleukin-16; Il-12p40, interleukin-12 subunit p40. The low R squared, in presence of both significant values, shows that even noisy, high-variability data can have a significant trend, even though data points fall further from the regression line in graph; d.v., dependent variable; i.v., independent variable. In bold are highlighted the significant ones.

Having found a link of IL-16 with both BMR and HGF, we asked ourselves whether IL- 16 could play an influencing role in the association between BMR and HGF, by adopting the extended regression, powerful technique to discover an endogenous variable. The statistical output confirmed the likely causal role of IL-16 concentrations, Table 6 .

Table 6. Evaluation of the influence of IL-16 in predicting BMR by HGF levels.

\begin{tabular}{|c|c|c|c|c|c|}
\hline \multicolumn{6}{|c|}{ Extended Linear Regression } \\
\hline \multirow[t]{2}{*}{ Number of Obs $=78$} & \multicolumn{2}{|c|}{$\begin{array}{c}\text { Wald Chi-Squared }(1)= \\
4.00\end{array}$} & \multicolumn{3}{|c|}{ Prob $>$ Chi-Squared $=0.005$} \\
\hline & Coef. & Std. Err. & $\mathrm{z}$ & $p>|z|$ & (95\% Conf. Interval) \\
\hline d.v. BMR & & & & & \\
\hline i.v. HGF & 3.114 & 1.558 & 2.00 & 0.046 & $0.061 / 6.168$ \\
\hline i.v. HGF & & & & & \\
\hline e.v IL-16 & 0.680 & 0.237 & 2.87 & 0.004 & $0.215 / 1.144$ \\
\hline $\operatorname{var}(\mathrm{e} . \mathrm{BMR})$ & 324139.1 & 164015.7 & - & - & $120232.0 / 873861.8$ \\
\hline $\operatorname{var}(\mathrm{e} . \mathrm{HGF})$ & 16214.72 & 2596.422 & - & - & $11847.0 / 22192.7$ \\
\hline $\begin{array}{l}\text { corr(e.HGF, } \\
\text { e.BMR)| }\end{array}$ & -0.6887355 & 0.1925947 & -3.58 & $<0.001$ & $-0.916 /-0.127$ \\
\hline
\end{tabular}

Legend to Table 6: BMR, basal metabolic rate, HGF, hepatic growth factor; IL-16, interleukin-16. The correlation estimates tells us about the endogeneity in our model of extended linear regression, i.e., IL-16 is an endogenous variable, playing likely a causal role. Although the Prob $>$ chi2 was significant due to the large coefficient, the F-statistic (Wald chi square) against the null, i.e., the excluded endogenous variable are irrelevant in the first-stage regression, should have been larger than 10 to demonstrate that instruments/covariate/endogenous/explanatory variables are weak according to Staiger and Stock's Rule of thumb; d.v., dependent variable; e.v., endogenous variable; i.v., independent variable. In bold are highlighted the significant ones and the correlation estimates.

Furthermore, having found a link of HGF with both BMR and SLD, we asked ourselves whether HGF concentrations, could play an influencing role in the association between BMR and the spleen length, evaluated as SLD, by using the same extended regression. The statistical output confirmed the likely causal role of HGF concentrations, Table 7. 
Table 7. Evaluation of the influence of HGF levels in predicting BMR by SLD.

\begin{tabular}{|c|c|c|c|c|c|}
\hline \multirow[t]{2}{*}{ Number of Obs $=78$} & \multicolumn{3}{|c|}{$\begin{array}{c}\text { Regression } \\
\text { Wald Chi-Squared (1) }=5.65\end{array}$} & \multicolumn{2}{|c|}{ Prob $>$ Chi-Squared $=0.017$} \\
\hline & Coef. & Std. Err. & $z$ & $p>|z|$ & (95\% Conf. Interval) \\
\hline $\begin{array}{c}\text { d.v. BMR } \\
\text { i.v. HGF } \\
\text { HGF }\end{array}$ & $\begin{array}{l}5.834 \\
0 \text { (omitted) }\end{array}$ & 2.457 & 2.38 & 0.017 & $1.022 / 10.653$ \\
\hline $\begin{array}{l}\text { i.v. HGF } \\
\text { e.v. SLD }\end{array}$ & 25.922 & 9.548 & 2.71 & 0.007 & $7.208-44.636$ \\
\hline $\begin{array}{l}\operatorname{var}(\mathrm{e} . \mathrm{BMR}) \\
\operatorname{var}(\mathrm{e} . \mathrm{HGF})\end{array}$ & $\begin{array}{l}729,014.7 \\
16,380.95\end{array}$ & $\begin{array}{l}499,010.4 \\
2622.96\end{array}$ & - & - & $\begin{array}{c}190,585.2 / 2,788,583.0 \\
11,968.58 / 22,420.0\end{array}$ \\
\hline $\begin{array}{c}\text { corr (e.HGF, } \\
\text { e.BMR) }\end{array}$ & -0.904 & 0.071 & -12.79 & $<0.001$ & $-0.978 / 0.627$ \\
\hline
\end{tabular}

Legend to Table 7: BMR, basal metabolic rate; SLD, spleen longitudinal diameter at ultrasonography; HGF, hepatic growth factor. The correlation estimates tells us about the endogeneity in our model of extended linear regression, i.e., HGF is an endogenous variable, playing likely a causal role. Although the Prob >chi2 was significant due to the large coefficient, the F-statistic (Wald chi square) against the null, i.e., that the excluded endogenous variable are irrelevant in the first-stage regression, should have been larger than 10 to demonstrate that instruments/covariate/endogenous/explanatory variables are weak according to Staiger and Stock's Rule of thumb; d.v., dependent variable; e.v., endogenous variable; i.v., independent variable. In bold are highlighted the significant ones and the correlation estimates.

Suffering the obese patients from HS, as per our selection, we tried to find some correlations between the severity of the liver fat, expressed as grades of HS, and spleen length, BMR and the two previously studied cytokines, i.e., IL-16 and IL-12p40. Only, SLD and BMR predicted HS at US, Table 8.

Table 8. Prediction of hepatic steatosis (HS) at US by SLD, BMR, IL-16 and IL-12p40.

\begin{tabular}{|c|c|c|c|c|c|}
\hline \multicolumn{6}{|c|}{ Ordered Probit Regression, Robust } \\
\hline Number of obs $=80$ & \multicolumn{5}{|c|}{ Pseudo $R$-squared $=0.148$} \\
\hline d.v. HS at US| & Coef. & Std. Err. & $z$ & $p>|z|$ & (95\% Conf. Interval) \\
\hline i.v. SLD & 0.428 & 0.095 & 4.48 & $<0.001$ & $0.241 / 0.615$ \\
\hline \multicolumn{6}{|c|}{ Ordered Probit Regression, Robust } \\
\hline Number of obs $=80$ & \multicolumn{5}{|c|}{ Pseudo $R$-squared $=0.112$} \\
\hline d.v. HS at US & Coef. & Std. Err. & $z$ & $p>|z|$ & (95\% Conf. Interval) \\
\hline i.v. BMR & 0.001 & 0.0003 & 3.86 & $<0.001$ & $0.0006 / 0.002$ \\
\hline \multicolumn{6}{|c|}{ Ordered Probit Regression, Robust } \\
\hline Number of obs $=78$ & \multicolumn{5}{|c|}{ Pseudo $R$-squared $=0.005$} \\
\hline d.v. HS at US & Coef. & Std. Err. & $z$ & $p>|z|$ & (95\% Conf. Interval) \\
\hline i.v. IL-16 & 0.002 & 0.002 & 1.00 & 0.318 & $0.002 / 0.005$ \\
\hline \multicolumn{6}{|c|}{ Ordered Probit Regression, Robust } \\
\hline Number of obs $=78$ & \multicolumn{5}{|c|}{ Pseudo $R$-squared $=0.004$} \\
\hline d.v. HS at US & Coef. & Std. Err. & $z$ & $p>|z|$ & (95\% Conf. Interval) \\
\hline i.v. IL-12p40 & -0.0007 & 0.0009 & -0.81 & 0.420 & $-0.002 / 0.001$ \\
\hline
\end{tabular}

At multiple linear regression, among SLD, BMR, IL-16 and IL-12p40, only SDL remained as predictor of HS severity with Coef., Std. Err., $z, p>|z|$, (95\% Conf. Interval) and Beta of 0.35; 12; 2.89; 0.004; $11-58$ and 36, respectively. The graph of this regression is shown in Figure 2. 


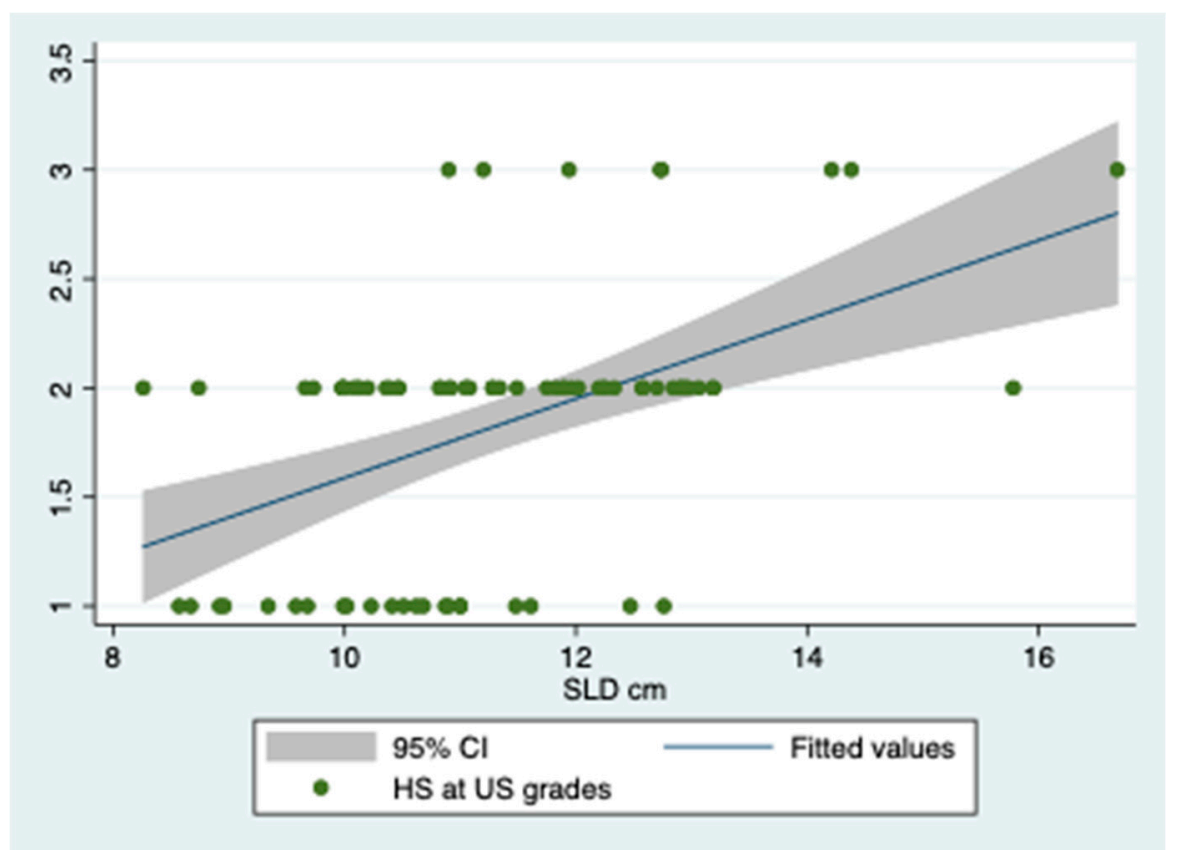

Figure 2. Association between spleen length and severity of hepatic steatosis. Legend to Figure 2: SLD, spleen longitudinal diameter; HS at US, hepatic steatosis at ultrasonography.

Coming back to the order probit regression, robust, evaluating the prediction of HS by SLD, inserting the interaction term, e.g., i.Gender\#c.HS, only HS of males was predicted by SLD $(t=2.55$, $p=0.014)$. Furthermore, inserting the interaction term, e.g., c.HS\#c.Age, the age of patients played a strong role in predicting HS by SLD $(t=4.83 ; p=0.000)$. Speculating on the role of BMR in predicting HS by SLD, we did not find that BMR was a mediator, Table 9.

Table 9. Evaluation of the influence of BMR in predicting HS by SLD.

\begin{tabular}{cccccc}
\hline \multirow{2}{*}{ Number of Obs $\mathbf{8 0}$} & \multicolumn{3}{c}{$\begin{array}{c}\text { Extended Linear Regression } \\
\text { Wald Chi-Sqaured (1) } \mathbf{1 6 . 2 1}\end{array}$} & \multicolumn{2}{c}{ Prob $>$ Chi-Squared $=\mathbf{0 . 0 0 0 1}$} \\
\hline & Coef. & Std. Err. & $z$ & $p>|z|$ & $(95 \%$ Conf. Interval) \\
\hline $\begin{array}{c}\text { d.v. HS at US } \\
\text { i.v. SLD } \\
\text { i.v SLD }\end{array}$ & $\begin{array}{c}0.308 \\
\text { (omitted) }\end{array}$ & 0.076 & $\mathbf{4 . 0 3}$ & $<0.001$ & $0.158 / 0.458$ \\
\hline $\begin{array}{c}\text { i.v. SLD } \\
\text { e.v. BMR }\end{array}$ & 0.002 & 0.0003 & $\mathbf{5 . 4 5}$ & $<0.001$ & $0.001 / 0.002$ \\
\hline var(e.HS) & 0.304 & 0.067 & - & - & $0.197 / 0.467$ \\
\hline
\end{tabular}

Legend to Table 9: HS at US, hepatic steatosis at ultrasonography; SLD, spleen longitudinal diameter; BMR, basal metabolic rate.

The lack of correlation estimates var (e.HS) tells us about the absence of endogeneity in our model of extended linear regression, i.e., BMR is not an endogenous variable, not playing likely a causal role. The Prob > chi2 was significant and the F-statistic (Wald chi square) against the null, i.e., the excluded endogenous variable are irrelevant in the first-stage regression, was larger than 10, demonstrating that instruments/covariate/endogenous/explanatory variables are weak according to Staiger and Stock's Rule of thumb; in our case is $<10$; d.v., dependent variable; e.v., endogenous variable; i.v., independent variable. It noticeable the absence of significance in the correlation estimates, although both the regressions were significant, indicating that the independent variable, i.e., SLD predicts the dependent variables, i.e., $\mathrm{HS}$ and BMR, independently one from the other. 
Having found that BMR does not influence the link between spleen volume and the grade of severity of HS, detected at ultrasonography, a successive step was to assess a possible association between spleen and HS studying other cytokines, which could play a significant role in modulating the interplay between this main immune organ and liver. The choice fell on IL-6, which demonstrated to be high influential, Table 10.

Table 10. Evaluation of the influence of Il-6 levels in predicting HS by SLD.

\begin{tabular}{|c|c|c|c|c|c|}
\hline \multirow[t]{2}{*}{ Number of Obs $=80$} & \multicolumn{3}{|c|}{$\begin{array}{l}\text { Extended Linear Regression. } \\
\text { Wald Chi-Squared }(1)=4.44\end{array}$} & \multicolumn{2}{|c|}{ Prob $>$ Chi-Squared $=0.035$} \\
\hline & Coef. & Std. Err. & $z$ & $p>|z|$ & (95\% Conf. Interval) \\
\hline \multicolumn{6}{|l|}{ d.v. HS at US } \\
\hline i.v. SLD & 7.437 & 3.528 & 2.11 & 0.035 & $0.522 / 14.353$ \\
\hline SLD & 0 (omitted) & & & & \\
\hline \multicolumn{6}{|l|}{ i.v. SLD } \\
\hline e.v. IL-6 & 0.0003 & 0.0004 & 0.71 & 0.479 & $-0.0005 /-0.001$ \\
\hline $\operatorname{var}(\mathrm{e} . \mathrm{HS})$ & 126.483 & 121.119 & - & - & $19.361 / 826.291$ \\
\hline $\operatorname{var}(\mathrm{e} . \mathrm{SLD})$ & 2.397 & 0.379 & - & - & $1.758 / 3.268$ \\
\hline \multicolumn{6}{|l|}{ corr(e.SLD) } \\
\hline (e.HS) & -0.9989576 & 0.000985 & -1014.18 & $<0.001$ & $-0.999 / 0.993$ \\
\hline
\end{tabular}

Legend to Table 10: The correlation estimates (e.SDL, e.HS) tells us about the endogeneity in our model of extended linear regression, i.e., IL-6 is an endogenous variable, playing likely a causal role. Although the Prob $>$ chi2 was significant due to the large coefficient, the F-statistic (Wald chi square) against the null, i.e., the excluded endogenous variable are irrelevant in the first-stage regression, should have been larger than 10 in order to demonstrate that instruments/covariate/endogenous/explanatory variables are weak according to Staiger and Stock's Rule of thumb; in our case is $<10$; d.v., dependent variable; e.v., endogenous variable; i.v., independent variable. In bold are highlighted the significant ones and the correlation estimates. It should be stressed the high value of $\mathrm{z}$ in the correlation (e.SLD, eHS).

Finally, to ascertain which among HGF, SLD, BMR and FFM predicted BARD score $>2$, a logistic regression was set, only BMR and FFM foreseeing the presence of advanced liver disease, evaluated by the afore mentioned score, Table 11.

Table 11. Prediction of hepatic steatosis or NAFLD severity using BARD score $>2$ by HGF, spleen length, indices of indirect calorimetry.

\begin{tabular}{|c|c|c|c|c|c|}
\hline Number of obs $=78$ & \multicolumn{4}{|c|}{ Logistic Regression } & Prob $>$ chi-squared $=0.244$ \\
\hline d.v. BARD & Odds Ratio & Std. Err. & $z$ & $p>|z|$ & (95\% Conf. Interval) \\
\hline i.v. HGF & 0.998 & 0.002 & -1.12 & 0.261 & $0.994 / 1.002$ \\
\hline \multicolumn{6}{|c|}{ Logistic Regression } \\
\hline Number of obs $=80$ & \multicolumn{5}{|c|}{ Prob $>$ chi-squared $=0.046$} \\
\hline d.v. BARD & Odds Ratio & Std. Err. & $z$ & $p>|z|$ & (95\% Conf. Interval) \\
\hline i.v. SLD & 0.730 & 0.121 & -1.89 & 0.058 & $0.527 / 1.011$ \\
\hline \multicolumn{6}{|c|}{ Logistic Regression } \\
\hline Number of obs $=80$ & \multicolumn{5}{|c|}{ Prob $>$ chi-squared $=0.023$} \\
\hline d.v. BARD & Odds Ratio & Std. Err. & $z$ & $p>|z|$ & (95\% Conf. Interval) \\
\hline i.v. BMR & 0.999 & 0.0006 & -2.14 & 0.032 & $0.997 / 0.999$ \\
\hline \multicolumn{6}{|c|}{ Logistic Regression } \\
\hline Number of obs $=80$ & \multicolumn{5}{|c|}{ Prob $>$ chi-squared $=0.019$} \\
\hline d.v. BARD & Odds Ratio & Std. Err. & $z$ & $p>|z|$ & (95\% Conf. Interval) \\
\hline i.v. FFM & 0.957 & 0.019 & -2.16 & 0.031 & $0.920 / 0.996$ \\
\hline
\end{tabular}

Legend to Table 11: HGF, hepatic growth factor; SLD, spleen longitudinal diameter; BMR, basal metabolic rate; d.v., dependent variable; i.v., independent variable. In bold are highlighted the significant ones. 
As collateral finding, a strong association was found firstly between HS and IMT, and secondly between HOMA values and HS at US, by linear regression (robust) with Coef., Std. Err., $t, p>|t|,(95 \%$ C.I.) $=0.017,0.005,3.15, \mathbf{0 . 0 0 2}, 0062,0.027$, respectively and by ordered profit regression (robust) with Coef. Std. Err., $z, p>|z|$, (95\% C.I): 0.13, 0.032, 4.02, 0.000, 0.066, 0.19, respectively.

Furthermore, a significant correlation was found between the VAT and IMT, as evident in the following results of linear regression (robust), Coef., Std. Err., $t, p>|t|,(95 \%$ C.I. $)=0.0045,0.0013,3.33$, $\mathbf{0 . 0 0 1}, 0.0018,0.0071$, respectlively.

The intra/inter-observational variability of US estimations was not significant, the mean difference being $1.9 \%, 2.2 \%$ and $3.1 \%$, and $3.9 \%, 3.3 \%$ and $3.4 \%$ for the HS, VAT and SLD, respectively, with a $\rho c$ of 0.91 .

The power of this research was carried out by measuring the difference of means and SDs of HGF- $\beta$ in the obese and controls groups, i.e., $501.46 \pm 134.75$ and $222.63 \pm 78.05$. The study turned out to be sufficiently powered (alpha $=0.001$, power $=0.95$ ) considering the sample size of the two studied populations (estimates sample size: total n 28, n 14 for group). Furthermore, the power analysis for a slope test in a simple linear regression, with a significant level of 0.001 and a power of 0.90 gave as estimated sample size $n 74$, using as the null slope the value of 0.4276 (Coefficient found in the regression: $\mathrm{SLD} \longrightarrow \mathrm{HS}$ ) and as the alternative one $=0$.

\section{Discussion}

In a retrospective way we studied the relationships between the energy expenditure of patients with obesity, likely characterised by a systemic, low-grade inflammatory process and some parameters of immune response, mediated by spleen, to try to explain the gap between HGF, increased in NAFLD patients and an early stage of cardiovascular disease, i.e., atherosclerosis. In this context, increased BMR may be a clue of MS [6] and NAFLD is a further expression of MS.

The core findings of this research could be summarised in: (j) HGF levels of the obese were higher than those of controls; (ji) BMR was foreseen by spleen length, which in turn was predicted by HGF; (ijj) BMR was predicted by IL-16, which predicted HGF; (jijj) FM predicted early atherosclerosis; (jijj) IL-12p40 did not predict IMT, HGF and BMR.

Interestingly, the BARD score $>2$ was negatively predicted by BMR and FFM. IL-16 was likely causal in predicting BMR by HGF levels. HGF was influential in predicting BMR by SLD level.

Still, HS was predicted by SLD in males of advanced age and by BMR. IL- 6 concentrations, but not BMR were influential in the prediction of HS by SLD.

Concerning the BARD score, only 36 patients (45\%) were classified as likely having the most severe form of NAFLD. Differently from NAFLD fibrosis score, this simple score bears the advantage of not ending up in a percentage of patients falling in the indeterminate category.

It is noticeable that the severity of HS at US in the obese was inversely associated with FFM hypothesising that exercise and rate of weight loss influence these patients.

What about the relationships to early atherosclerosis, evaluated as IMT? IMT, among the afore mentioned cytokines, IL-16 and IL-12p40, the spleen length and indirect calorimetry indices, was associated only with FM, according to numerous studies linking adiposity, mainly abdominal, with a high cardiovascular risk.

It is found that excessive adipose tissue mass (FM) as well as a (partial) lack of adipose tissue predisposes towards chronic cardio-metabolic diseases. This implies that total adipose tissue mass is not the predominant factor that explains the increased metabolic risk in obese individuals. Rather, the location where the excessive calories are stored in combination with adipose tissue function seem to determine metabolic health [40]. Also HGF is not related to IMT, reinforcing the finding that this growth factor as well as spleen play a role in the advanced stage of cardiovascular disease and not in the early one.

Unexpectedly, we found a correlation between HGF and IL-2 receptor-alpha as well as between HGF and MIP-1b (data not shown), but their significance should be further deepened. 
How do our results fit with existing literature? In a study of 28 patients researchers found that serum HGF levels were higher in NASH patients than in the controls, although it was statistically insignificant and a correlation with MS could not be detected [24]. Our data are in discordance with the previously mentioned research, because there was no significant difference of HGF levels between the more and less severe form of HS (performed on BARD score $>2$ ). Nevertheless, we stress that HGF levels predicted both severity of HS at US and spleen length that is a clue for advanced NAFLD.

Indeed, other pieces of evidence are steadily accumulating, in the sense that HGF induces an antioxidant response in hepatic cells, countering oxidative stress, main component of NAFLD [41]. Furthermore, deletion of HGF (hepatocyte growth factor)/mesenchymal-epithelial transition factor (c-Met) receptor leads to the development of severe NASH in mice [42]. Finally, HGF has a key role in insulin resistance, mechanism central to NAFLD onset and progression [43].

On the contrary, in mice HGF improves IR and prevents high-fat-diet-induced obesity [44]. Trying to explain the causal role of IL-16 in predicting BMR by HGF levels, we draw the attention on pathological conditions having overlapping mechanisms with NAFLD. Authors found that IL-16 levels significantly correlate to the disease activity of psoriasis, the duration of which is associated to spleen volume $[45,46]$. IL-16 might play an important role in the inflammatory process of patients suffering from acute myocardial infarction and correlates with inflammatory cell activation [47]. IL-16 is constitutively present in peripheral blood monocytes and spontaneously released during apoptosis [48].

About IL-12p40, we found no significant associations with early atherosclerosis and/or the studied parameter of immune system, i.e., HGF and/or the BMR. Vice versa, having evaluated the influence of spleen, assessed as SLD, in predicting BMR by HGF levels, we confirm the role of this organ in relation to energy expenditure and immune system.

As final consideration, the energy expenditure, observed in obese patients as a consequence of a systemic, low-grade, inflammatory process, may explain progression from obesity to MS, independent of the presence of NAFLD. I should point out that excess adiposity per se, even without accompanying metabolic health status, may contribute to fibrosis progress [49]. Recent data confirm that both FFM and FM are significant contributors to BMR [50].

\section{Limitations}

The findings in the current analysis nevertheless had a variety of caveats of their own, including the fact that, due to the characteristics of prediction (its bi-directionality), we do not know for sure which is the cause or the effect but this intertwined link provides a thought-provoking example of how apparently different biological properties could interact to determine a unique abnormal condition of health. Liver biopsy to assess the severity of NAFLD was nor originally performed for ethical reasons (absence of frank cytolysis in the obese) and technical difficulty due to the abundant adipose tissue. Notably, because of the retrospective nature of the study, we analysed only the available data. Finally, these data should be confirmed by prospective studies.

\section{Conclusions}

We have found a link between BMR, spleen volume and HGF levels in patients with obesity-related NAFLD. Noticeably, spleen as an immune organ, even though it plays a role in advanced cardiovascular disease, was of scarce impact in the subclinical stage of atherosclerosis.

Regardless, there is a long road ahead in demonstrating how HGF works in relation to the spleen, and there is much to understand what and how this growth factor is linked to BMR. That said, the strides in immunology in recent years make it tempting to speculate that growth factors may play an increasing role in modifying the progression of some diseases.

\section{Future Directions}

As with much obesity research these days, the study raises more questions than it does answers, and it is not entirely clear how these specific mechanisms can be harnessed into a useful clinical 
treatment. We suggest that whether these mechanisms were identified, researchers could potentially hone in on specific human health outcomes influencing them. From that point, future treatments could conceivably modulate serum cytokines, growth factors to benefit human health.

Author Contributions: G.T. conceived the study, carried out the statistics and wrote the manuscript. V.C. contributed to gathering data and analysing them. P.C. contributed to gathering data. C.B. contributed to analysing results and gave criticism to the research. D.C. contributed to drafting the manuscript, analysing results and gave criticism to the research.

Conflicts of Interest: The authors declare no conflict of interest.

\section{References}

1. Chawla, A. The immune system as a sensor of the metabolic state. Immunity 2013, 8, 644-654.

2. Ravussin, E.; Lillioja, S.; Knowler, W.C.; Christin, L.; Freymond, D.; Abbott, W.G.; Boyce, V.; Howard, B.V.; Bogardus, C. Reduced rate of energy expenditure as a risk factor for body-weight gain. N. Engl. J. Med. 1988, 318, 467-472. [CrossRef] [PubMed]

3. Carneiro, I.P.; Elliott, S.A.; Siervo, M.; Padwal, R.; Bertoli, S.; Battezzati, A.; Prado, C.M. Is Obesity Associated with Altered Energy Expenditure? Adv. Nutr. 2016, 7, 476-487. [CrossRef] [PubMed]

4. Lazzer, S.; Bedogni, G.; Lafortuna, C.L.; Marazzi, N.; Busti, C.; Galli, R.; De Col, A.; Agosti, F.; Sartorio, A. Relationship between basal metabolic rate, gender, age, and body composition in 8,780 white obese subjects. Obesity 2010, 18, 71-78. [CrossRef] [PubMed]

5. Bouchard, C.; Blair, S.N. Introductory comments for the consensus on physical activity and obesity. Med. Sci. Sports Exerc. 1999, 31, S498-S501. [CrossRef] [PubMed]

6. Tarantino, G.; Marra, M.; Contaldo, F.; Pasanisi, F. Basal metabolic rate in morbidly obese patients with non-alcoholic fatty liver disease. Clin. Investig. Med. 2008, 3, E24-E29. [CrossRef]

7. De Aquino, E.A., Jr.; Carbinatto, F.M.; Moriyama, L.T.; Bagnato, V.S. Regression of Non-Alcoholic Fatty Liver by Metabolic Reduction: Phototherapy in Association with Aerobic Plus Resistance Training In Obese Man-A Pilot Study. J. Obes. Weight Loss Ther. 2018, 8, 61.

8. Javed, F.; He, Q.; Davidson, E.L.; Thornton, C.J.; Albu, J.; Boxt, L.; Krasnow, N.; Elia, M.; Kang, P.; Heshka, S.; et al. Brain and high metabolic rate organ mass: Contributions to resting energy expenditure beyond fat-free mass. Am. J. Clin. Nutr. 2010, 91, 907-912. [CrossRef] [PubMed]

9. Altunkaynak, B.Z.; Ozbek, E.; Altunkaynak, M.E. A stereological and histological analysis of spleen on obese female rats, fed with high fat diet. Saudi Med. J. 2007, 28, 353-357. [PubMed]

10. Benter, T.; Klühs, L.; Teichgräber, U. Sonography of the spleen. J. Ultrasound Med. 2011, 30, 1281-1293. [CrossRef]

11. Kim, E.J.; Kim, S.; Kang, D.O.; Seo, H.S. Metabolic activity of the spleen and bone marrow in patients with acute myocardial infarction evaluated by $18 \mathrm{f}$-fluorodeoxyglucose positron emission tomograpic imaging. Cardiovasc. Imaging 2014, 7, 454-560. [CrossRef] [PubMed]

12. Dutta, P.; Courties, G.; Wei, Y.; Leuschner, F.; Gorbatov, R.; Robbins, C.S.; Iwamoto, Y.; Thompson, B.; Carlson, A.L.; Heidt, T.; et al. Myocardial infarction accelerates atherosclerosis. Nature 2012, 487, 325-329. [CrossRef] [PubMed]

13. Swirski, F.K.; Nahrendorf, M.; Etzrodt, M.; Wildgruber, M.; Cortez-Retamozo, V.; Panizzi, P.; Figueiredo, J.L.; Kohler, R.H.; Chudnovskiy, A.; Waterman, P.; et al. Identification of splenic reservoir monocytes and their deployment to inflammatory sites. Science 2009, 325, 612-616. [CrossRef] [PubMed]

14. Emami, H.; Singh, P.; MacNabb, M.; Vucic, E.; Lavender, Z.; Rudd, J.H.; Fayad, Z.A.; Lehrer-Graiwer, J.; Korsgren, M.; Figueroa, A.L.; et al. Splenic metabolic activity predicts risk of future cardiovascular events: Demonstration of a cardiosplenic axis in humans. JACC Cardiovasc. Imaging 2015, 8, 121-130. [CrossRef] [PubMed]

15. Tarantino, G.; Colicchio, P.; Conca, P.; Finelli, C.; Di Minno, M.N.; Tarantino, M.; Capone, D.; Pasanisi, F. Young adult obese subjects with and without insulin resistance: What is the role of chronic inflammation and how to weigh it non-invasively? J. Inflamm. 2009, 6, 6. [CrossRef] [PubMed]

16. Tarantino, G.; Conca, P.; Pasanisi, F.; Ariello, M.; Mastrolia, M.; Arena, A.; Tarantino, M.; Scopacasa, F.; Vecchione, R. Could inflammatory markers help diagnose nonalcoholic steatohepatitis? Eur. J. Gastroenterol. Hepatol. 2009, 21, 504-511. [CrossRef] [PubMed] 
17. Suzuki, S.; Yamanouchi, K.; Soeta, C.; Katakai, Y.; Harada, R.; Naito, K.; Tojo, H. Skeletal muscle injury induces hepatocyte growth factor expression in spleen. Biochem. Biophys. Res. Commun. 2002, 292, 709-714. [CrossRef] [PubMed]

18. Kono, S.; Nagaike, M.; Matsumoto, K.; Nakamura, T. Marked induction of hepatocyte growth factor induction mRNA in intact kidney and spleen in response to injury of distant organs. Biochem. Biophys. Res. Commun. 1992, 186, 991-998. [CrossRef]

19. Skibinski, G.; Skibinska, A.; James, K. The role of hepatocyte growth factor and its receptor c-met in interactions between lymphocytes and stromal cells in secondary human lymphoid organs. Immunology 2001, 102, 506-514. [CrossRef]

20. Yang, Z.J.; Zhang, Y.R.; Chen, B.; Zhang, S.L.; Jia, E.Z.; Wang, L.S.; Zhu, T.B.; Li, C.J.; Wang, H.; Huang, J.; et al. Phase I clinical trial on intracoronary administration of Ad-hHGF treating severe coronary artery disease. Mol. Biol. Rep. 2009, 36, 1323-9132. [CrossRef]

21. Hahn, W.; Pyun, W.B.; Kim, D.S.; Yoo, W.S.; Lee, S.D.; Won, J.H.; Shin, G.J.; Kim, J.M.; Kim, S. Enhanced cardioprotective effects by coexpression of two isoforms of hepatocyte growth factor from naked plasmid DNA in a rat ischemic heart disease model. J. Gene. Med. 2011, 13, 549-555. [CrossRef] [PubMed]

22. Komarowska, I.; Coe, D.; Wang, G.; Haas, R.; Mauro, C.; Kishore, M.; Cooper, D.; Nadkarni, S.; Fu, H.; Steinbruchel, D.A.; et al. Hepatocyte Growth Factor Receptor c-Met Instructs T Cell Cardiotropism and Promotes T Cell Migration to the Heart via Autocrine Chemokine Release. Immunity 2016, 42, 1087-1099. [CrossRef] [PubMed]

23. Rychli, K.; Richter, B.; Hohensinner, P.J.; Kariem Mahdy, A.; Neuhold, S.; Zorn, G.; Berger, R.; Mörtl, D.; Huber, K.; Pacher, R.; et al. Hepatocyte growth factor is a strong predictor of mortality in patients with advanced heart failure. Heart 2011, 97, 1158-1163. [CrossRef] [PubMed]

24. Balaban, Y.H.; Sumer, H.; Simsek, H.; Us, D.; Tatar, G. Metabolic syndrome, non-alcoholic steatohepatitis (NASH), and hepatocyte growth factor (HGF). Ann. Hepatol. 2006, 5, 109-114. [CrossRef]

25. Lichtenauer, M.; Franz, M.; Fritzenwanger, M.; Figulla, H.R.; Gerdes, N.; Jung, C. Elevated plasma levels of interleukin-12p40 and interleukin-16 in overweight adolescents. Biomed. Res. Int. 2015, 2015, 940910. [CrossRef] [PubMed]

26. Chupp, G.L.; Wright, E.A.; Wu, D.; Vallen-Mashikian, M.; Cruikshank, W.W.; Center, D.M.; Kornfeld, H.; Berman, J.S. Tissue and T cell distribution of precursor and mature IL-16. J. Immunol. 1998, 161, 3114-3119.

27. Jana, M.; Pahan, K. IL-12 p40 homodimer, but not IL-12 p70, induces the expression of IL-16 in microglia and macrophages. Mol. Immunol. 2009, 46, 773-783. [CrossRef] [PubMed]

28. Cooper, A.M.; Khader, S.A. IL-12p40: An inherently agonistic cytokine. Trends Immunol. 2007, 28, 33-38. [CrossRef]

29. The International Committee of Medical Journal Editors (ICMJE) at Overlapping Publication. Available online: http://www.icmje.org/recommendations/browse/publishing-and-editorial-issues/overlappingpublications.html (accessed on 27 August 2019).

30. Tarantino, G.; Costantini, S.; Finelli, C.; Capone, F.; Guerriero, E.; La Sala, N.; Gioia, S.; Castello, G. Carotid Intima-Media Thickness Is Predicted by Combined Eotaxin Levels and Severity of Hepatic Steatosis at Ultrasonography in Obese Patients with Nonalcoholic Fatty Liver Disease. PLoS ONE 2014, 9, e105610. [CrossRef]

31. Matthews, D.R.; Hosker, J.P.; Rudenski, A.S.; Naylor, B.A.; Treacher, D.F.; Turner, R.C. Homeostasis model assessment: Insulin resistance and beta-cell function from fasting plasma glucose and insulin concentrations in man. Diabetologia 1985, 28, 412-419. [CrossRef]

32. Harrison, S.A.; Oliver, D.; Arnold, H.L.; Gogia, S.; Neuschwander-Tetri, B.A. Development and validation of a simple NAFLD clinical scoring system for identifying patients without advanced disease. Gut 2008, 57, 1441-1447. [CrossRef] [PubMed]

33. Webb, M.; Yeshua, H.; Zelber-Sagi, S.; Santo, E.; Brazowski, E.; Halpern, Z.; Oren, R. Diagnostic value of a computerized hepatorenal index for sonographic quantification of liver steatosis. AJR Am. J. Roentgenol. 2009, 192, 909-914. [CrossRef] [PubMed]

34. Tarantino, G.; Costantini, S.; Finelli, C.; Capone, F.; Guerriero, E.; La Sala, N.; Gioia, S.; Castello, G. Is serum Interleukin-17 associated with early atherosclerosis in obese patients? J. Transl. Med. 2014, 12, 214. [CrossRef] [PubMed] 
35. Stein, J.H.; Korcarz, C.E.; Hurst, R.T.; Lonn, E.; Kendall, C.B.; Mohler, E.R.; Najjar, S.S.; Rembold, C.M.; Post, W.S. American Society of Echocardiography Carotid Intima-Media Thickness Task Force. Use of carotid ultrasound to identify subclinical vascular disease and evaluate cardiovascular disease risk: A consensus statement from the American Society of Echocardiography Carotid Intima-Media Thickness Task Force. Endorsed by the Society for Vascular Medicine. J. Am. Soc. Echocardiogr. 2008, 21, 93-111. [PubMed]

36. Schutz, Y.; Kyle, U.U.G.; Pichard, C. Fat-free mass index and fat mass index percentiles in caucasians aged 18-98 y. Int. J. Obes. 2002, 26, 953-960. [CrossRef] [PubMed]

37. What Is Difference between Endogeneous and Exogeneous Variables? Available online: http://wwwpersonal. umd.umich.edu/ \{\}delittle/Encyclopedia\%20entries/Endogenous\%20variable.htm (accessed on 27 August 2019).

38. Normal Physiological Levels of Human Cytokines Using Bio-Plex Pro ${ }^{\mathrm{TM}}$ Cytokine Assays. Available online: http://www.bio-rad.com/webroot/web/pdf/lsr/literature/Bulletin_6029.pdf (accessed on 27 August 2019).

39. Lee, J.K.; Shim, J.H.; Lee, H.C.; Lee, S.H.; Kim, K.M.; Lim, Y.S.; Chung, Y.H.; Lee, Y.S.; Suh, D.J. Estimation of the healthy upper limits for serum alanine aminotransferase in Asian populations with normal liver histology. Hepatology 2010, 51, 1577-1583. [CrossRef]

40. Goossens, G.H. The Metabolic Phenotype in Obesity: Fat Mass, Body Fat Distribution, and Adipose Tissue Function. Obes. Facts. 2017, 10, 207-215. [CrossRef]

41. Domínguez-Pérez, M.; Nuño-Lámbarri, N.; Clavijo-Cornejo, D.; Luna-López, A.; Souza, V.; Bucio, L.; Miranda, R.U.; Muñoz, L.; Gomez-Quiroz, L.E.; Uribe-Carvajal, S.; et al. Hepatocyte Growth Factor Reduces Free Cholesterol-Mediated Lipotoxicity in Primary Hepatocytes by Countering Oxidative Stress. Oxid. Med. Cell Longev. 2016, 2016, 7960386. [CrossRef]

42. Kroy, D.C.; Schumacher, F.; Ramadori, P.; Hatting, M.; Bergheim, I.; Gassler, N.; Boekschoten, M.V.; Müller, M.; Streetz, K.L.; Trautwein, C. Hepatocyte specific deletion of c-Met leads to the development of severe non-alcoholic steatohepatitis in mice. J. Hepatol. 2014, 61, 883-890. [CrossRef]

43. Oliveira, A.G.; Araújo, T.G.; Carvalho, B.M.; Rocha, G.Z.; Santos, A.; Saad, M.J.A. The Role of Hepatocyte Growth Factor (HGF) in Insulin Resistance and Diabetes. Front. Endocrinol. 2018, 9, 503. [CrossRef]

44. Muratsu, J.; Iwabayashi, M.; Sanada, F.; Taniyama, Y.; Otsu, R.; Rakugi, H.; Morishita, R. Hepatocyte Growth Factor Prevented High-Fat Diet-Induced Obesity and Improved Insulin Resistance in Mice. Sci. Rep. 2017, 7, 130. [CrossRef] [PubMed]

45. Purzycka-Bohdan, D.; Szczerkowska-Dobosz, A.; Zablotna, M.; Wierzbicka, J.; Piotrowska, A.; Zmijewski, M.A.; Nedoszytko, B.; Nowicki, R. Assessment of Interleukin 16 Serum Levels and Skin Expression in Psoriasis Patients in Correlation with Clinical Severity of the Disease. PLoS ONE 2016, 11, e0165577. [CrossRef]

46. Balato, N.; Napolitano, M.; Ayala, F.; Patruno, C.; Megna, M.; Tarantino, G. Nonalcoholic fatty liver disease, spleen and psoriasis: New aspects of low-grade chronic inflammation World. J. Gastroenterol. 2015, 21, 6892-6897. [CrossRef] [PubMed]

47. Schernthaner, C.; Paar, V.; Wernly, B.; Pistulli, R.; Rohm, I.; Jung, C.; Figulla, H.R.; Yilmaz, A.; Cadamuro, J.; Haschke-Becher, E.; et al. Elevated plasma levels of interleukin-16 in patients with acute myocardial infarction. Medicine 2017, 96, e8396. [CrossRef] [PubMed]

48. Elssner, A.; Doseff, A.I.; Duncan, M.; Kotur, M.; Wewers, M.D. IL-16 is constitutively present in peripheral blood monocytes and spontaneously released during apoptosis. J. Immunol. 2004, 172, 7721-7725. [CrossRef] [PubMed]

49. Kim, Y.; Chang, Y.; Cho, Y.K.; Ahn, J.; Shin, H.; Ryu, S. Metabolic healthy versus unhealthy obesity and risk of fibrosis progression in non-alcoholic fatty liver disease. Liver Int. 2019. 21 June Epub ahead of print. [CrossRef]

50. Johnstone, A.M.; Murison, S.D.; Duncan, J.S.; Rance, K.A.; Speakman, J.R. Factors influencing variation in basal metabolic rate include fat-free mass, fat mass, age, and circulating thyroxine but not sex, circulating leptin, or triiodothyronine. Am. J. Clin. Nutr. 2005, 82, 941-948. [CrossRef]

(C) 2019 by the authors. Licensee MDPI, Basel, Switzerland. This article is an open access article distributed under the terms and conditions of the Creative Commons Attribution (CC BY) license (http://creativecommons.org/licenses/by/4.0/). 